\title{
BOUNDED MULTIPLIER CONVERGENCE IN MEASURE OF RANDOM VECTOR SERIES
}

\section{RYLL-NARDZEWSKI AND W. A. WOYCZYŃSKI}

ABSTRACT. If the series $\Sigma f_{i}$ of random vectors with values in a $\mathrm{Ba-}$ nach space converges unconditionally in measure, then, for each $\left(\lambda_{i}\right) \in l^{\infty}$, the series $\Sigma \lambda_{i} f_{i}$ also converges unconditionally in measure.

Recall that the series of elements of a topological group is said to be unconditionally convergent if it remains convergent after arbitrary permutation of its terms. The following proposition is, essentially, a classical result due to Orlicz (cf. also [2, Theorem 1]).

Proposition 1. Let $\left(x_{i}\right)$ be a sequence of elements of a sequentially complete topological abelian group. Then the series $\Sigma x_{i}$ converges unconditionally if and only if for every sequence of $\epsilon_{i}= \pm$ the series $\Sigma \epsilon_{i} x_{i}$ converges.

Now, let $(X,\|\cdot\|)$ be a Banach space and let $(T, \mathcal{A}, \mu)$ be a probability system. By definition $L^{0}(T, \mathbb{A}, \mu ; \mathfrak{X})=L^{0}(\mathfrak{X})$ is the linear complete metric space of all measurable functions on $(T, \mathscr{A}, \mu)$ with values in $\mathcal{X}$ (i.e. $\mu$-a.e. limits of simple functions) equipped with the quasi-norm

$$
\|f\|_{0}=\min \left\{c: c^{-1} \mu\{t:\|f(t)\|>c\} \leq 1\right\} .
$$

Theorem 1. If for all choices of $\epsilon_{i}= \pm 1$ the series $\Sigma \epsilon_{i} f_{i}, f_{i} \in L^{0}(X)$, converges in $L^{0}(\mathcal{X})$ then also for each sequence $\left(\lambda_{i}\right) \in l^{\infty}$ the series $\Sigma \lambda_{i} f_{i}$ converges in $L^{0}(X)$.

In the special case $X=R$ this theorem was obtained by Maurey and $\mathrm{Pi}$ sier [1] but our method of proof is much shorter and more elementary. Proposition 1 and Theorem 1 give us immediately the following theorem which confirms a long-standing conjecture (cf. [3, Problem III.6.8]).

Theorem 2. In $L^{0}(\mathcal{X})$, unconditional convergence of the series $\Sigma f_{i}$ is equivalent to convergence of all the series $\Sigma \lambda_{i} f_{i},\left(\lambda_{i}\right) \in l^{\infty}$.

Notice that Theorem 2 may be used as a tool in constructing an integral of any bounded real measurable function with respect to the bounded (random) measure with values in $L^{0}(\mathcal{X})$ (cf. [3, Theorem III.6.2]). For integrals with

Received by the editors July $11,1974$.

AMS (MOS) subject classifications (1970). Primary 46E40; Secondary $46 \mathrm{G} 10$. vector.

Key words and phrases. Unconditional convergence, bounded multiplier, random 
respect to random measures with independent values on disjoint sets more information is available from [4].

Proof of Theorem 1. By [2, Lemma 2], given the sequence $\lambda=\left(\lambda_{i}\right),\left|\lambda_{i}\right|$ $\leq 1$, for each sequence $\left(x_{i}\right) \subset \mathcal{X}$ and arbitrary $n \in N$, we have the inequality

$$
P\left\{\left(\epsilon_{i}\right):\left\|\sum_{i=1}^{n} \epsilon_{i} x_{i}\right\|>\frac{1}{8}\left\|\sum_{i=1}^{n} \lambda_{i} x_{i}\right\|\right\} \geq \frac{1}{8},
$$

where $P=\left(P_{0}+P_{\lambda}\right) / 2$, and $P_{\lambda}$ is the probability on $\{-1,+1\}^{N}$ such that the random variables $\{-1,+1\}^{\mathbf{N}} \ni\left(\epsilon_{i}\right) \rightarrow \epsilon_{j}, j=1,2, \cdots$, are independent and $\int \epsilon_{j} d P_{\lambda}=\lambda_{j}$. Now, given the sequence $\left(f_{i}\right) \subset L^{0}(\mathcal{X})$, for each $t \in T$, we have that

$$
P\left\{\left(\epsilon_{i}\right):\left\|\sum_{i=1}^{n} \epsilon_{i} f_{i}(t)\right\|>\frac{1}{8}\left\|\sum_{i=1}^{n} \lambda_{i} f_{i}(t)\right\|\right\} \geq \frac{1}{8}, \quad n \in \mathbf{N} .
$$

Integrating this inequality with respect to the measure $\mu$ we get that

$$
(\mu \times P)\left\{\left(t,\left(\epsilon_{i}\right)\right):\left\|\sum_{i=1}^{n} \epsilon_{i} f_{i}(t)\right\|>\frac{1}{8}\left\|\sum_{i=1}^{n} \lambda_{i} f_{i}(t)\right\|\right\} \geq \frac{1}{8}, \quad n \in \mathbf{N} .
$$

By the Fubini theorem (notice that max is taken over the finite set)

$$
\max _{\left(\epsilon_{i}\right)} \mu\left\{t:\left\|\sum_{i=1}^{n} \epsilon_{i} f_{i}(t)\right\|>\frac{1}{8}\left\|\sum_{i=1}^{n} \lambda_{i} f_{i}(t)\right\|\right\} \geq \frac{1}{8}, \quad n \in \mathbf{N} .
$$

Now, let

$$
T_{1} \stackrel{\mathrm{df}}{=}\left\{t: \frac{1}{8}\left\|\sum_{i=1}^{n} \lambda_{i} f_{i}(t)\right\|>c\right\}, \quad c>0,
$$

and $\mu\left(T_{1}\right)>0$. Put $\mu_{1}(A) \stackrel{\text { df }}{=} \mu\left(A \cap T_{1}\right) / \mu\left(T_{1}\right), A \in \mathbb{A}$. Applying (1) to the measure $\mu_{1}$ (instead of $\mu$ ) we have that

$$
\begin{aligned}
\underset{\left(\epsilon_{i}\right)}{\max _{1}} \mu_{1}\left\{t:\left\|\sum_{i=1}^{n} \epsilon_{i} f_{i}(t)\right\|>c\right\} \\
\geq \max _{\left(\epsilon_{i}\right)} \mu_{1}\left\{t:\left\|\sum_{i=1}^{n} \epsilon_{i} f_{i}(t)\right\|>\frac{1}{8}\left\|\sum_{i=1}^{n} \lambda_{i} f_{i}(t)\right\|\right\} \geq \frac{1}{8}, \quad n \in \mathbf{N},
\end{aligned}
$$

and, eventually, by definition of $\mu_{1}$, we get that

(2) $\max _{\left(\epsilon_{i}\right)} \mu\left\{t:\left\|\sum_{i=1}^{n} \epsilon_{i} f_{i}(t)\right\|>c\right\} \geq \frac{1}{8} \mu\left\{t:\left\|\sum_{i=1}^{n} \lambda_{i} f_{i}(t)\right\|>8 c\right\}, \quad n \in \mathbf{N}$,

which concludes the proof of Theorem 1 . 
Notice that inequality (2) implies that for each $c>0$

$$
\begin{aligned}
\left\{c: \frac{1}{c} \max _{\left(\epsilon_{i}\right)} \mu\{t\right. & \left.\left.:\left\|\sum_{i=1}^{n} \epsilon_{i} f_{i}(t)\right\|>c\right\} \leq 1\right\} \\
& \subset\left\{\frac{1}{3} c: \frac{1}{c} \mu\left\{t:\left\|\sum_{i=1}^{n} \lambda_{i} f_{i}(t)\right\|>c\right\} \leq 1\right\},
\end{aligned}
$$

and that the above inclusion and the definition of the quasi-norm $\|\cdot\|_{0}$ give us also the following quantitative result.

Theorem 3. Let $\left(f_{i}\right) \subset L^{0}(\mathfrak{X})$ and $\left(\lambda_{i}\right) \in l^{\infty},\left|\lambda_{i}\right| \leq 1$. Then for each $n \in \mathbf{N}$

$$
\left\|\sum_{i=1}^{n} \lambda_{i} f_{i}\right\|_{0} \leq 3 \max _{\left(\epsilon_{i}\right)}\left\|\sum_{i=1}^{n} \epsilon_{i} f_{i}\right\|_{0} .
$$

\section{REFERENCES}

1. B. Maurey and G. Pisier, Un théorème d'extrapolation et ses consequences, C. R. Acad. Sci. Paris Sér. A-B 277 (1973), A39-A42.

2. K. Musial, C. Ryll-Nardzewski and W. A. Woyczyński, Convergence presque surs des séries aléatoires vectorielles à multiplicateur bornée, C. R. Acad. Sci. Paris Sér. A-B 279 (1974), A225-A228.

3. S. Rolewicz, Metric linear spaces, PWN, Warsaw, 1972.

4. K. Urbanik and W. A. Woyczyński, A random integral and Orlicz spaces, Bull. Acad. Polon. Sci. Sér. Sci. Math. Astronom. Phys. 15 (1967), 161-169. MR 35 \#6170.

INSTITUTE OF MATHEMATICS, WROCLAW UNIVERSITY, PL. GRUNWALDZKI 2/4, 50384 WROCLAW, POLAND

INSTITUTE OF MATHEMATICS, POLISH ACADEMY OF SCIENCES, PL. GRUNWALDZKI 2/4, 50384 WROCLAW, POLAND 